\title{
Semi-Synchronous Transductions
}

\author{
Vince Bárány
}

Received: date / Accepted: date

\begin{abstract}
Semi-synchronously rational relations generalise synchronised rational relations in a natural way. We discuss here some of their basic properties, among them a "Cobham-Semenov-like" dichotomy theorem. Our main result is a characterisation of bijective semi-synchronously rational transductions as those bijections mapping regular relations to regular ones and non-regular relations to non-regular ones.
\end{abstract}

Keywords formal languages · rational transductions · semi-synchronous relations · regularity-preserving mappings

\section{Introduction}

A relation on words is semi-synchronously rational if it is accepted by an asynchronous multi-tape finite automaton processing each tape at a prescribed pace. Semi-synchronously rational relations thus constitute a natural generalisation of the synchronised rational relations studied in [9]. The notion is briefly mentioned in [17] but only to raise a very natural question, which was subsequently answered independently in [1] and in [7]. This result showing that, except in trivial cases, the relative speed of reading individual tapes is uniquely determined, is presented here in Theorem 1.

In the context of symbolic dynamics semi-synchronous (sequential) transductions (on (bi-)infinite words) are natural devices in transforming dynamical systems [3]. Our initial interest stems from the world of automatic presentations, where our main result, Theorem 2, spells out that bijective semi-synchronous transductions (on finite words) are the natural model of translations among equivalent automatic presentations of infinite structures [1].

Work done while affiliated with RWTH Aachen and partially supported by the project ANR06-MDCA DocFlow.

V. Bárány

LaBRI, Université Bordeaux 1

351 cours de la Libération, F-33405 Talence cedex

E-mail: vbarany@logic.rwth-aachen.de 
In [11] Maurer and Nivat studied bijective rational transductions. They have shown that there is a rational bijection between two regular languages if and only if these have the same asymptotic growth: either both are exponentially growing or both grow at a polynomial rate of the same degree or both of them are finite and of the same size.

Recently, Béal, Lombardy, and Sakarovitch [2] proved that there is always a letterto-letter rational bijection between any two regular languages having exactly the same number of elements of every length.

While growth arguments do play a key role in our analysis, we present here a characterisation of semi-synchronously rational bijections primarily in terms of preservation of regularity of relations. This is an essential distinction from works on regularitypreserving [14] or continuous transductions [15] that are concerned with mappings of words preserving the regularity of languages, i.e. unary relations, under taking images or pre-images.

In the course of our investigation we make two further observations along these lines. Proposition 4 and Theorem 3 assert that every bijective transduction that maps every regular binary relation to a regular one is in fact semi-synchronously rational, provided that it is length-preserving (in which case it is synchronised rational), respectively, that its domain is a regular language of exponential density.

\section{Semi-synchronously rational relations}

Let $\Sigma$ be a finite alphabet. The length of a word $w \in \Sigma^{*}$ is denoted by $|w|$, the empty word by $\varepsilon$, and for each $0<i \leq|w|$ the $i$ th symbol of $w$ is written as $w[i]$. We consider relations on words, i.e. subsets $R$ of $\left(\Sigma^{*}\right)^{n}$ for some $n>0$. Asynchronous $n$-tape automata accept precisely the rational relations, i.e., rational subsets of the product monoid $\left(\Sigma^{*}\right)^{n}$. Finite transducers are asynchronous 2-tape automata and the relations they recognise are commonly referred to as rational transductions [4]. A relation $R \subseteq$ $\left(\Sigma^{*}\right)^{n}$ is synchronised rational [9], or simply regular, if it is accepted by a synchronous $n$-tape automaton. We introduce the following generalisation.

Definition 1 (Semi-synchronously rational relations)

Let $\square$ be a special end-marker symbol, $\square \notin \Sigma$, and $\Sigma_{\square}=\Sigma \cup\{\square\}$. Further let $\alpha=\left(a_{1}, \ldots, a_{n}\right)$ be a vector of positive integers and consider a relation $R \subseteq\left(\Sigma^{*}\right)^{n}$. The $\alpha$-convolution of $R$ is the set $\bigotimes_{\alpha} R=\left\{\left(w_{1} \square^{m_{1}}, \ldots, w_{n} \square^{m_{n}}\right) \mid\left(w_{1}, \ldots, w_{n}\right) \in R\right.$ and the $m_{i}$ are minimal such that there is a $k$ with $k a_{i}=\left|w_{i}\right|+m_{i}$ for every $\left.i\right\}$. This allows us to identify $\otimes_{\alpha} R$ with a subset of the monoid $\left(\left(\Sigma_{\square}\right)^{a_{1}} \times \cdots \times\left(\Sigma_{\square}\right)^{a_{n}}\right)^{*}$. If $\bigotimes_{\alpha} R$ thus corresponds to a regular set, then we say that $R$ is $\alpha$-synchronously rational, or just $\alpha$-synchronous. Finally, $R$ is semi-synchronous if it is $\alpha$-synchronous for some $\alpha$.

Intuitively, our definition expresses that although $R$ requires an asynchronous automaton to accept it, synchronicity can be regained when processing words in blocks, the size of which are component-wise fixed by $\alpha$. As a special case, for $\alpha=\mathbf{1}$, we obtain the regular relations.

Example 1 Consider $R=\left\{\left(a^{n}, a^{2 n+1}\right) \mid n \in \mathbb{N}\right\}$. While $R$ is not synchronised rational, $\bigotimes_{(1,2)} R=(a, a a)^{*}(\square, a \square)$ is. Hence, $R$ is $(1,2)$-synchronous.

Also note that for every $\alpha=\left(a_{1}, \ldots, a_{n}\right)$ the convolution $\otimes_{\alpha}\left(\left(\Sigma^{*}\right)^{n}\right)$ is the regular subset of $\left(\left(\Sigma_{\square}\right)^{a_{1}} \times \cdots \times\left(\Sigma_{\square}\right)^{a_{n}}\right)^{*}$ of all words whose end markers are lined up at the end of each component consistently with Definition 1 . 
Recall that a relation $R \subseteq\left(\Sigma^{*}\right)^{n}$ is recognisable if it is saturated by a congruence (of the product monoid $\left(\Sigma^{*}\right)^{n}$ ) of finite index, equivalently, if it is a finite union of direct products of regular languages [9]. We denote by Rat, SRat, $\mathrm{S}_{\alpha}$ Rat, Reg, Rec the classes of rational, semi-synchronous, $\alpha$-synchronous, regular, and recognisable relations respectively.

It is a straightforward consequence of the definition that for any fixed $\alpha$ the class of $\alpha$-synchronously rational relations has all the convenient properties of synchronised rational relations.

Proposition $1 \mathrm{~S}_{\alpha}$ Rat is an effective boolean algebra for each $\alpha$. The projection of every $\alpha \beta$-synchronous relation onto the first $|\alpha|$ many components, is $\alpha$-synchronous.

Proof One applies the classical automata constructions for taking products, determinising and complementing finite automata over the alphabet $\left(\Sigma_{\square}\right)^{a_{1}} \times \cdots \times\left(\Sigma_{\square}\right)^{a_{n}}$. For sound treatment of the end-markers complements have to be taken relative to the regular domain $\bigotimes_{\alpha}\left(\left(\Sigma^{*}\right)^{n}\right)$ of those words actually representing the convolution of some tuple.

Proposition 2 For every vector $\alpha$ of non-negative integers, $\mathrm{S}_{\alpha}$ Rat is closed under taking images (hence also inverse images) via semi-synchronous transductions.

Proof Let $T$ be a $(p, q)$-synchronous transduction, $R$ an $\alpha$-synchronous $n$-ary relation with $\alpha=\left(a_{1}, \ldots, a_{n}\right)$. Then $T(R)=\left\{\mathbf{v} \mid \exists \mathbf{u} \in R, \forall i \leq n:\left(u_{i}, v_{i}\right) \in T\right\}$ is the projection of the $\left(q a_{1}, \ldots, q a_{n}, p a_{1}, \ldots, p a_{n}\right)$-synchronous relation $\{(\mathbf{v}, \mathbf{u}) \mid \mathbf{u} \in R, \forall i \leq$ $\left.n:\left(u_{i}, v_{i}\right) \in T\right\}$. Therefore, by Proposition $1, T(R)$ is $\left(q a_{1}, \ldots, q a_{n}\right)$-synchronously rational, hence also $\alpha$-synchronously rational (cf. Theorem 1(i) below). Closure under taking inverse images follows from the fact that the inverse of a $(p, q)$-synchronous transduction is $(q, p)$-synchronous.

Observe that the composition of a $(p, q)$-synchronous and an $(r, s)$-synchronous transduction is $(p r, q s)$-synchronous, thus, the class of semi-synchronous transductions is closed under composition.

Next we show that for $(p, q)$-synchronously rational transductions, with the exception of recognisable transductions, the ratio $p / q$ is uniquely determined. This is a crucial property that can be helpful in showing that certain relations are not semisynchronous.

To this end let us say that $\alpha$ and $\beta$ are dependent if $k \cdot \alpha=l \cdot \beta$ for some $k, l \in \mathbb{N}$, where multiplication is meant component-wise. Then, comparing classes $\mathrm{S}_{\alpha}$ Rat and $\mathrm{S}_{\beta}$ Rat we observe the following "Cobham-Semenov-like" relationship. Theorem 1 provides solution to [17, Probléme 6.3] and has been independently proved by the author [1] and by Carton [7].

Theorem 1 Let $n, p, q \in \mathbb{N}$ and $\alpha, \beta \in \mathbb{N}^{n}$.

(i) If $\alpha$ and $\beta$ are dependent, then $\mathrm{S}_{\alpha}$ Rat $=\mathrm{S}_{\beta}$ Rat.

(ii) If $(p, q)$ and $(r, s)$ are independent, then $\mathrm{S}_{(p, q)} \operatorname{Rat} \bigcap \mathrm{S}_{(r, s)}$ Rat $=\operatorname{Rec}$.

Proof

(i) Clearly, a relation $R$ is $\alpha$-synchronous if and only if it is $(k \cdot \alpha)$-synchronous for any $k \geq 1$. The claim follows.

(ii) Recognisable relations are trivially $\alpha$-synchronous for any $\alpha$, therefore we only care for the other inclusion. 
Let $R \in \mathrm{S}_{(p, q)}$ Rat $\bigcap \mathrm{S}_{(r, s)}$ Rat. We need to show that $R$ is a finite union of Cartesian products $A_{i} \times B_{i}$ of regular languages. Consider the following equivalence relation on words in the domain of $R$ :

$$
x \sim x^{\prime} \stackrel{\text { def }}{\Longleftrightarrow} \forall y: R(x, y) \leftrightarrow R\left(x^{\prime}, y\right)
$$

By Proposition 1 this is a regular relation. Each equivalence class $[x]$ is therefore a regular language. Similarly, for every $x$ the set $x R=\{y \mid(x, y) \in R\}$ is regular. Hence, $R$ is of the (irreducible) form $\bigcup_{i=1}^{r} A_{i} \times B_{i}$ if and only if there are precisely $r$ equivalence classes $\left[x_{1}\right], \ldots,\left[x_{r}\right]$ modulo $\sim$, in which case $A_{i}=\left[x_{i}\right]$ and $B_{i}=x_{i} R$ for $1 \leq i \leq r$.

According to (i), $R$ is both ( $p r, q r)$ - and ( $p r, p s)$-synchronous, and by assumption $p s \neq q r$, wlog. $p s<q r$. Let us further assume for simplicity and wlog. that $p r=1$ and let $k=p s$ and $l=q r$. Consider some FDA's $\mathcal{A}$ and $\mathcal{A}^{\prime}$ recognising $\bigotimes_{(1, k)} R$ and $\bigotimes_{(1, l)} R$, respectively. As transducers, $\mathcal{A}$ is thus "slower" then $\mathcal{A}^{\prime}$ in reading the second tape. Let $C=|\mathcal{A}|^{2}+1$, where $|\mathcal{A}|$ is the number of states of $\mathcal{A}$. The following observation is confirmed by a straightforward pumping argument:

$$
x \not x^{\prime} \Rightarrow \exists y:|y|<k\left(\max \left(|x|,\left|x^{\prime}\right|\right)+C\right) \wedge R(x, y) \leftrightarrow \neg R\left(x^{\prime}, y\right)
$$

The syntactic congruence of $\mathcal{A}^{\prime}$ induces an equivalence of finite index on pairs of words $(u, z) \in\left(\Sigma \cup\{\square\} \times(\Gamma \cup\{\square\})^{l}\right)^{*}$, i.e. $(u, z) \approx_{\mathcal{A}^{\prime}}\left(u^{\prime}, z^{\prime}\right)$ iff their actions on the states of $\mathcal{A}^{\prime}$ are identical. Let $K$ be the length of the longest word $v$ such that $\left(v, \square^{l|v|}\right)$ is the shortest such representant of its $\approx_{\mathcal{A}^{\prime} \text {-class. }}$

Consider now any $x$ long enough such that $\left\lceil(|x|+C) \frac{k}{\tau}\right\rceil+K<|x|$. During the run of $\mathcal{A}^{\prime}$ on input $(x, y)$ for any $y$ shorter than $k(|x|+C), y$ will be completely read leaving a suffix $v$ of $x, v$ longer than $K$, unread. By replacing $v$ with a shorter $v^{\prime}$ such that $\left(v, \square^{l|v|}\right) \approx \mathcal{A}^{\prime}\left(v^{\prime}, \square^{l\left|v^{\prime}\right|}\right)$ in $x$ we obtain an $x^{\prime}$ shorter than $x$, such that, by $\left(^{*}\right)$, $x \sim x^{\prime}$. Thus we have shown that each $\sim$-class has a representant of bounded size, i.e. that there are finitely many such classes as required.

In $[9$, Theorem 5.1] it has been established that Rec $\subsetneq$ Reg $\subsetneq$ DRat $\subsetneq$ Rat, where DRat refers to the class of deterministic rational relations. All regular relations are easily seen to be deterministic rational $[9$, Prop. 5.2] and this naturally extends to semisynchronously rational relations. By definition, for every $R \in \mathrm{S}_{\alpha}$ Rat its $\alpha$-convolution $\bigotimes_{\alpha} R$ is a regular language, which can thus be accepted by a DFA. It is then straightforward to transform this DFA into a deterministic transducer accepting $R$. Hence we have that Reg $\subset$ SRat $\subset$ DRat. In fact, both inclusions are strict: the relation $\left\{\left(a^{n}, a^{2 n}\right) \mid n \in \mathbb{N}\right\}$ is evidently $(1,2)$-synchronous but not $(1,1)$-synchronously rational, and we have the following corollary of Theorem 1 .

Corollary 1 The relation $\left\{\left(a^{n}, a^{2 n}\right),\left(b^{n}, b^{3 n}\right) \mid n \in \mathbb{N}\right\}$ is deterministic rational but not semi-synchronous. Hence SRat $\subsetneq$ DRat and SRat is not closed under union.

Proof Let $R_{a}=\left\{\left(a^{n}, a^{2 n}\right) \mid n \in \mathbb{N}\right\}$ and $R_{b}=\left\{\left(b^{n}, b^{3 n}\right) \mid n \in \mathbb{N}\right\}$. These are $(1,2)$-synchronously rational and $(1,3)$-synchronously rational, respectively, and their union $R$ is obviously deterministic rational. Assuming $R$ to be $(p, q)$-synchronous both $R_{a}=R \cap a^{*} \times a^{*}$ and $R_{b}=R \cap b^{*} \times b^{*}$ would also have to be $(p, q)$-synchronous by Proposition 1 . However, since neither $R_{a}$ nor $R_{b}$ is recognisable, according to Theorem 1 we would have $1 / 2=p / q=1 / 3$, which is impossible. 
The problem of whether a given rational relation is synchronised rational is known to be undecidable $[4,9]$. Simple reductions show that the same holds true for the question of semi-synchronicity.

Proposition 3 For any given $p, q \in \mathbb{N}$ the following problems are undecidable.

(i) Given a rational transduction $R \in$ Rat is $R \in \mathrm{S}_{(p, q)}$ Rat?

(ii) Given a rational transduction $R \in$ Rat is $R \in \mathrm{SRat}$ ?

Proof Problem (i) is equivalent to the problem of deciding regularity of rational relations over the alphabet $\left(\Sigma_{\square}^{p} \times \Sigma_{\square}^{q}\right)$ that is known to be undecidable [4,9].

To prove (ii) consider the following reduction. ${ }^{1}$ Given a rational transduction $R \subseteq$ $\left(\Sigma^{*}\right)^{2}$ let $R^{\prime}=R \uplus R^{-1}$ where $R^{-1}$ is understood to be over a disjoint copy of $\Sigma$. Clearly, if $R$ is regular, then so is $R^{\prime}$. Conversely, if $R^{\prime}$ is $(p, q)$-synchronously rational then, by Proposition 1 , both $R=R^{\prime} \cap\left(\Sigma^{*} \times \Sigma^{*}\right)$ and, similarly, $R^{-1}$ are $(p, q)$ synchronously rational. However, the latter implies that $R$ is also $(q, p)$-synchronously rational. Then, according to Theorem 1 , either $p=q$ or $R$ is recognisable. In either case, $R$ is regular. Therefore a decision procedure for (ii) would yield an algorithm for regularity, which is undecidable.

\section{Bijective semi-synchronous transductions}

The objects of study in this section are translations.

Definition 2 (Translations) A translation is a bijection $t: D \rightarrow C$ between regular sets of words $D \subseteq \Sigma^{*}$ and $C \subseteq \Gamma^{*}$. A translation $t$ preserves regularity (non-regularity) if the image of every regular relation under $t$ (respectively, under $t^{-1}$ ) is again regular. Finally, $t$ is weakly regular if it preserves both regularity and non-regularity.

Although every bijective rational transduction qualifies as a translation, in fact one preserving regularity of sets, it is not necessarily regularity preserving.

\section{Example 2}

- The homomorphism $w \mapsto \bar{w}$ with domain $\{a, b\}^{*}$ and $\bar{a}=b$ and $\bar{b}=a$ is a translation that is regularity preserving and is its own inverse.

- The homomorphism dupl mapping $a \mapsto a a$ for every $a \in \Sigma$ is a $(1,2)$-synchronous transduction, thus, by Proposition 2, it is weakly regular.

- Consider the mapping bin : $a^{n} \mapsto[n]_{2}$, where $[n]_{2}$ is the binary numeral representing $n$ in the least-significant digit first manner. It is well known that such binary representation $[R]_{2}=\left\{\left(\left[n_{1}\right]_{2}, \ldots,\left[n_{r}\right]_{2}\right) \mid\left(n_{1}, \ldots, n_{r}\right) \in R\right\}$ of every Presburgerdefinable relation $R \subseteq \mathbb{N}^{r}$ is synchronised rational [6], and that regularity over unary numerals implies Presburger definability ${ }^{2}$. It follows that bin is a regularity preserving translation. However, the pre-image $\operatorname{bin}^{-1}\left(\left\{\left[2^{n}\right]_{2} \mid n \in \mathbb{N}\right\}\right)$ is clearly not a regular subset of $a^{*}$.

\footnotetext{
1 I thank the anonymous referee suggesting a similar reduction.

2 The following stronger statement is a classical one (see e.g. [13]). The unary representation of a relation $R \subseteq \mathbb{N}^{r}$ is synchronised rational iff $R$ is first-order definable in the structure $\left(\mathbb{N}, 0,<,+1,\{\equiv \bmod (m)\}_{m>1}\right)$, in which case it is even quantifier-free definable.
} 
- The homomorphism $\tau$ mapping $a \mapsto a a$ and $b \mapsto b$ is a rational translation that is not regularity preserving, e.g. $\tau\left(\left\{\left(a^{n}, b^{n}\right) \mid n \in \mathbb{N}\right\}\right)$ is not regular.

- rev : $w \mapsto w^{\text {rev }}$ mapping each word to its reversal is a translation which preserves regularity of sets, i.e. unary relations, in both directions but the image of the prefix relation under reversal is not a regular relation as the reader can check.

- The injection $w \mapsto w w$ with domain $\{a, b\}^{*}$ is not a translation because its range is not regular.

Regular translations, whose graphs are synchronised rational relations, are easily seen to be weakly regular. More generally, like dupl above, all semi-synchronous transductions are weakly regular as we have already established in Proposition 2.

Corollary 2 Every semi-synchronously rational translation is weakly regular.

As our main result, in Theorem 2 we prove the converse of this statement. The proof relies on a careful analysis of growth rates and on the regularity of (pre)images of certain distinguished relations. We divide the proof into several steps with the aid of equivalent transformations. By this we mean the following. Given a translation $f: D \rightarrow C$ and a weakly regular translation $T: C \rightarrow C^{\prime}$ we "replace" $f$ with $g=T \circ f: D \rightarrow C^{\prime}$. We will say that two translations $f: D \rightarrow C$ and $g: D \rightarrow C^{\prime}$ over the same domain are equivalent $(f \sim g)$ if one can be obtained from the other by a transformation as above, that is, if $g \circ f^{-1}$ is weakly regular. As far as preserving regularity of relations is concerned this is a perfectly legitimate transformation, because for each relation $R$ over $D, f(R)$ is regular if and only if $g(R)$ is regular; and, equivalently, for each relation $R$ over $C^{\prime}, f^{-1}\left(T^{-1}(R)\right)$ is regular if and only if $g^{-1}(R)$ is regular. In particular, whenever $f \sim g$ then $f$ preserves regularity, or non-regularity, or is weakly regular if and only if $g$ does/is, respectively.

\subsection{Growth}

To each translation $f: D \rightarrow C$ we associate its growth function $G_{f}: \mathbb{N} \rightarrow \mathbb{N}$ defined as

$$
G_{f}(n)=\max (\{|f(u)|: u \in D,|u| \leq n\} \cup\{0\}) \quad \text { for each } n .
$$

We define the following growth-related properties of a translation $f$ :

- $f$ is length-preserving if $|f(x)|=|x|$ for every word $x$;

- $f$ is length-monotonic if $|x| \leq|y|$ implies $|f(x)| \leq|f(y)|$ for every $x$ and $y$;

- $f$ has bounded delay ${ }^{3}$ if there exists a constant $\delta$

such that $|x|+\delta<|y|$ implies $|f(x)|<|f(y)|$ for every $x$ and $y$.

We recall some basic combinatorial facts concerning regular sets and relations. The first one is a straightforward consequence of the well-known "pumping lemma" of automata theory. A relation $R$ of arity $n+m$ is locally finite if for every $\left(x_{1}, \ldots, x_{n}\right)$ there are only finitely many $\left(y_{1}, \ldots, y_{m}\right)$ such that $R(\mathbf{x}, \mathbf{y})$ holds.

Fact $3([8])$ Let $R \subseteq\left(\Sigma^{*}\right)^{n+m}$ be a regular and locally finite relation. Then there is a constant $k$ such that $\max _{j}\left|y_{j}\right| \leq \max _{i}\left|x_{i}\right|+k$ holds for every $(\mathbf{x}, \mathbf{y}) \in R$. In particular, if $f:\left(\Sigma^{*}\right)^{n} \rightarrow \Sigma^{*}$ is a regular function, then there is a constant $k$ such that for every $\mathbf{x}$ in its domain we have $|f(\mathbf{x})| \leq \max _{i}\left|x_{i}\right|+k$.

\footnotetext{
3 Different from [9]'s notion of rational transducers having bounded delay.
} 
For a regular set $D \subseteq \Sigma^{*}$ let $D=n=D \cap \Sigma^{n}$ and $D_{\leq n}=D \cap \Sigma^{\leq n}$ denote the set of members of $D$ of length precisely $n$ and at most $n$, respectively. Further let $\operatorname{Pref}(D)$ be the (regular) set of prefixes of words in $D$.

Fact 4 ([10, Lemma 3.12]) Let $D \subseteq \Sigma^{*}$ be a regular set. Then

(i) $\left|\operatorname{Pref}(D)_{=n}\right|=\mathcal{O}\left(\left|D_{<n}\right|\right)$ and

(ii) for every fixed $C \in \mathbb{N}^{\prime}:\left|D_{\leq(n+C)}\right|=\mathcal{O}\left(\left|D_{\leq n}\right|\right)$

The density function $d$ of a regular language $D$ maps each natural $n$ to the number $\left|D_{\leq n}\right|$ of elements of $D$ of length at most $n$. Concerning the density functions of regular languages we recall the following facts.

Fact $5([\mathbf{1 8}, \mathbf{1 9}])$ Let $D$ be an infinite regular language and $d(n)$ its density function. Then either

(i) $D$ is the finite union of languages $D_{i}=u_{i, 1} v_{i, 1}^{*} u_{i, 2} \ldots u_{i, n_{i}} v_{i, n_{i}}^{*} u_{i, n_{i}+1}$ and $d(n)$ is a polynomial of degree $\max _{i} n_{i}$, or

(ii) there are $\alpha>1$ and $r \in \mathbb{N}$ and constants $0<c_{1} \leq c_{2}$, such that $c_{1} n^{r} \alpha^{n} \leq d(n) \leq$ $c_{2} n^{r} \alpha^{n}$ for all sufficiently large $n$.

Proof

The first assertion is a well-known characterisation of regular languages with polynomial density, cf. e.g. [19]. We outline a proof of (ii) based on classical results of [18].

Consider the sequence $\left(\left|\operatorname{Pref}(D)_{n}\right|\right)_{n}$. Because $\operatorname{Pref}(D)$ is prefix closed, i.e. all states of its minimal DFA are accepting, the sequence $\left(\left|\operatorname{Pref}(D)_{n}\right|\right)_{n}$ is a D0L sequence, and, as such, it has a definite asymptotic behaviour. Indeed, for every D0L sequence $s$ not ultimately zero there are $\alpha \geq 1$ and $r \in \mathbb{N}$, such that $s$ has growth order $n^{r} \alpha^{n}$, i.e. $s(n)=\Theta\left(n^{r} \alpha^{n}\right)$. [18, Section III.7.]. In our case now $\alpha>1$. Moreover, the sequence $\left(\left|\operatorname{Pref}(D)_{\leq n}\right|\right)_{n}$, which is the summation of the former, is itself a D0L sequence of the same growth order, because $n^{r} \alpha^{n} \leq \sum_{i=0}^{n} i^{r} \alpha^{i} \leq n^{r} \sum_{i=0}^{n} \alpha^{i}<\frac{\alpha}{\alpha-1} n^{r} \alpha^{n}$. To conclude we note that by Fact 4 there is a constant $C>0$ such that $C \cdot\left|\operatorname{Pref}(D)_{n n}\right| \leq\left|D_{\leq n}\right|$ and obviously $\left|D_{\leq n}\right| \leq\left|\operatorname{Pref}(D)_{\leq n}\right|$. Therefore also $d(n)=\Theta\left(n^{r} \alpha^{n}\right)$ as claimed.

The relation $\mathbb{L}=\{(x, y)|| y|\leq| x \mid\}$ will play a central role in our analysis. We leave the domain of $\mathbb{L}$ intentionally unspecified and rather overload the symbol $\mathbb{L}$ to allow it to refer to $\left\{(x, y) \in D^{2}|| y|\leq| x \mid\right\}$ for any regular language $D$ relevant in the particular context.

Our first lemma establishes a connection between regularity of the pre-image of $\mathbb{L}$ under a translation and its property of having bounded delay.

Lemma 1 Let $f: D \rightarrow C$ be a translation such that $f^{-1}(\mathbb{L})$ is regular. Then $f$ has bounded delay.

Proof By assumption $f^{-1}(\mathbb{L})$ is regular and it is locally finite because $\mathbb{L}=\{(u, v) \in$ $\left.C^{2}|| v|\leq| u \mid\right\}$ is. Hence, by Fact 3 , there is a constant $\delta$ such that $\left|f^{-1}(v)\right| \leq$ $\left|f^{-1}(u)\right|+\delta$ whenever $|v| \leq|u|$. By negating both sides and substituting $u=f(x)$ and $v=f(y)$ we arrive at the equivalent statement that $|y|>|x|+\delta$ implies that $|f(y)|>|f(x)|$, which is to say that $f$ has bounded delay with bound $\delta$.

The following lemma gives a handy example of an equivalent transformation that will also be the first step in our construction. 
Lemma 2 To every translation $f: D \rightarrow C$ for which $f(\mathbb{L})$ is regular one can construct an equivalent translation $g=\pi \circ f$, such that $\pi$ is regular, $G_{g}=G_{f}, g$ is lengthmonotonic, moreover $g$ has bounded delay iff $f$ has bounded delay.

Proof The relation $\mathbb{L}=\left\{(x, y) \in D^{2}|| y|\leq| x \mid\right\}$ is locally finite and regular, so is its image $f(\mathbb{L})$. Therefore, by Fact 3 , there is a constant $K$ such that $|y| \leq|x| \rightarrow|f(y)| \leq$ $|f(x)|+K$ for every $x, y \in D$. By the choice of $K$, we have $G_{f}(|x|) \leq|f(x)|+K$ for all $x \in D$. We may thus partition $D$ into subsets

$$
D_{s}=\left\{x \in D\left|G_{f}(|x|)-\right| f(x) \mid=s\right\} \text { with } 0 \leq s \leq K .
$$

We claim that the sets $C_{s}=f\left(D_{s}\right)$ constitute a regular partitioning of $C$. Indeed, consider the sets

$$
F_{s}=\{u \in C|\forall v \in C:(u, v) \in f(\mathbb{L}) \rightarrow| v|\leq| u \mid+s\}
$$

for all $0 \leq s \leq K$. These are regular, being first-order definable from $f(\mathbb{L})$, which is by assumption regular, and from the relation $|v| \leq|u|+s$, which is trivially regular for any fixed $s$. Then $C_{0}=F_{0}$ and $C_{s+1}=F_{s+1} \backslash F_{s}$ for all $0 \leq s<K$, therefore $C_{s}$ is regular for each $s$ as claimed. This observation allows us to define $g$ by padding the $f$-image of each word according to its partition:

$$
g(x)=f(x) @^{G_{f}(|x|)-|f(x)|} \quad(\forall x \in D)
$$

Thus, $g(D)=C^{\prime}=\bigcup_{s=1}^{k} C_{s} \cdot @^{s}$ and $g=\pi \circ f$, where $\pi$ is the padding function defined as $\pi=\left\{(v, w) \in C \times C^{\prime} \mid \bigvee_{s \leq K}\left(v \in C_{s} \wedge w=v @^{s}\right)\right\}$. Given automata recognising each $C_{s}$ it is again easy to construct a synchronised rational transducer recognising $\pi$, which is thus (weakly) regular. This means that $g$ is equivalent to $f$ as required. Moreover, $g$ is length-monotonic by construction, because $|g(x)|=G_{f}(|x|)=G_{g}(|x|)$ holds for every word $x$, and the growth function $G_{f}$ is by definition always monotonic.

To check our last claim assume first that $f$ has bounded delay, say bounded by $\delta$. Thus, $|x|+\delta<|y| \Longrightarrow|f(x)|<|f(y)|$ for every $x$ and $y$, and therefore $|g(x)|=$ $G_{f}(|x|)<|f(y)| \leq|g(y)|$ whenever $|x|+\delta<|y|$, i.e. $g$ has bounded delay. Conversely, assume that $g$ has bounded delay, say bounded by $\Delta$. We shall assume that $D$ is infinite, otherwise the claims of this lemma are rendered trivial. We may then choose $M$ such that for every $n$ there is a word $z \in D$ of length $n \leq|z|<n+M$. Let $x, y \in D$ be arbitrary words such that $|x|+K(\Delta+M)<|y|$. Then, by the choice of $M$ we have for each $i \leq K$ some word $x_{i} \in D$ with $x_{0}=x$ and $\left|x_{i}\right|+\Delta<\left|x_{i+1}\right| \leq\left|x_{i}\right|+(\Delta+M)$ for all $i<K$ and $\left|x_{K}\right|<|y|$. Thus $|g(x)|<\left|g\left(x_{1}\right)\right|<\ldots<\left|g\left(x_{K}\right)\right| \leq|g(y)|$, so $|g(x)|+K<|g(y)|$, in other terms $\left|G_{f}(x)\right|+K<\left|G_{f}(y)\right|$. Therefore $|f(x)|+K \leq$ $\left|G_{f}(x)\right|+K<\left|G_{f}(y)\right| \leq|f(y)|+K$, i.e. $|f(x)|<|f(y)|$. Since the choice of $x$ and $y$ was arbitrary, this proves that $f$ has bounded delay, with $\delta=K(\Delta+M)$ as a bound.

The next observation further highlights the significance of preserving regularity of $\mathbb{L}$ and of the bounded delay property. The following crucial fact will be key to our proof of the main theorem.

Lemma 3 Let $f: D \rightarrow C$ be a translation of bounded delay for which $f(\mathbb{L})$ is regular. Then the infinite sequence of increments $\partial G_{f}=\left\langle G_{f}(1)-G_{f}(0), G_{f}(2)-G_{f}(1), \ldots\right\rangle$ of the growth function of $f$ is ultimately periodic. 
Proof Relying on Lemma 2 we assume henceforth that $f$ is length-monotonic. We know that $f$ has bounded delay, say with bound $\delta$. Then $G_{f}$ is a non-decreasing sequence of naturals in which each number can occur at most $\delta$ times.

Let $\leq_{\text {llex }}$ denote the length-lexicographical ordering, and let $E=\left\{(x, y) \in D^{2} \mid\right.$ $|x|=|y|\}$. Both $\leq_{\text {llex }}$ and $f(E)$ are regular relations. The latter so, because $(x, y) \in$ $E \leftrightarrow(x, y),(y, x) \in \mathbb{L}$ and $f(\mathbb{L})$ is regular by assumption. We define the language

$$
\begin{aligned}
L & =\left\{f(x)|x \in D, \forall y \in D| x|=| y \mid \rightarrow f(x) \leq_{\text {llex }} f(y)\right\} \\
& =\left\{u \in C \mid \forall v \in C(u, v) \in f(E) \rightarrow u \leq_{\text {llex }} v\right\}
\end{aligned}
$$

which is thus also regular. Let $l_{0}<l_{1}<\ldots$ be the sequence of all those naturals $l$ for which there is a word in $D$ of length $l$. Then $L=\left\{u_{0}, u_{1}, \ldots\right\}$, where $u_{i}$ denotes, for each $i \in \mathbb{N}$, the length-lexicographically least element of $f\left(D_{=l_{i}}\right)$. Because $f$ is lengthmonotonic, we have $\left|u_{i}\right|=G_{f}\left(l_{i}\right)$ and $\left|u_{i}\right| \leq\left|u_{i+1}\right|$ for each $i \in \mathbb{N}$. Furthermore, by the choice of $\delta$, also $\left|u_{i}\right|<\left|u_{i+\delta}\right|$ holds for each $i \in \mathbb{N}$. In other words, there are at most $\delta$ many words in $L$ of each length. One says that $L$ is $\delta$-thin [16]. We can thus write $L$ as a disjoint union of the regular languages

$$
L_{k}=\left\{u \in L\left|\exists^{=k} v \in L:\right| u|=| v \mid\right\} \quad(1 \leq k \leq \delta)
$$

Let $\psi: C \rightarrow a^{*}$ be the homomorphism sending every letter to $a$. It is a lengthpreserving projection mapping $L$ to the set of unary numerals corresponding to the pruned sequence obtained from $G_{f}$ by omitting the repetitions, i.e. $\psi(L)$ is the unary representation of $G_{f}(\mathbb{N})$. Similarly, for each $1 \leq k \leq \delta, \psi\left(L_{k}\right)$ is the unary representation of the set of those values $n$ that are repeated exactly $k$ times in $G_{f}$, i.e. such that $\left|G_{f}^{-1}(n)\right|=k$.

As homomorphic images of regular languages these projections are regular unary languages. As such, each $\psi\left(L_{k}\right)$ is the unary representation of a semi-linear set of naturals $N_{k}=\left\{n_{k, 0}<n_{k, 1}<n_{k, 2}<\ldots\right\}$, which is to say that the sequences $\left(n_{k, i+1}-\right.$ $\left.n_{k, i}\right)_{i}$ are ultimately periodic. In particular they are bounded, say by $B$. One way to conclude is by building a finite sequential transducer $T$ which on input $a^{\omega}$ outputs $\partial G_{f}$. We can construct $T$ from a direct product of automata recognising $\psi\left(L_{1}\right), \ldots, \psi\left(L_{\delta}\right)$ and a counter counting modulo $B+1$. The counter value is initially 0 and is incremented upon reading each $a$ while the component automata are simulated. Whenever one of the automata enters an accepting state, the counter value is output and reset to 0 . If it was the $k+1$-st automaton, then additionally $k$ zeros are appended to the output. By definition, $\partial G_{f}$ is the output of the infinite run on input $a^{\omega}$. Equivalently, $\partial G_{f}$ is the homomorphic image of the ultimately periodic sequence of states along this run. The claim follows.

Corollary 3 If $f$ is a translation such that both $f(\mathbb{L})$ and $f^{-1}(\mathbb{L})$ are regular then $\partial G_{f}$ is ultimately periodic.

In our last lemma of this subsection we show how the fact that the growth function of a translation increases in periodic increments can be exploited to transform it into an equivalent length-preserving translation.

Lemma 4 Let $g: D \rightarrow C$ be a length-monotonic regularity-preserving translation of bounded delay. Then one can construct an equivalent length-preserving translation $h=\tau \circ g$ with $\tau$ a semi-synchronous transduction. 
Proof Under the assumptions on $g$ Lemma 3 shows that $\partial G_{g}$ is ultimately periodic. For simplicity we assume wlog. that $G_{g}(0)=0$, i.e. that if $\varepsilon \in D$ then $g(\varepsilon)=\varepsilon$. (This can be ensured by modifying $g$, when needed, on a finite number of words, which always yields an equivalent translation with the same asymptotic properties.) This allows us to construct a length-preserving translation $h$ equivalent to $g$ by subdividing words in the image of $g$ into blocks according to $\partial G_{g}$.

To this end let $c=\max _{n} \partial G_{g}[n]$ and consider a new alphabet $\Theta=\Gamma^{\leq c}=\{w \in$ $\left.\Gamma^{*}:|w| \leq c\right\}$. Let $\beta: \Theta^{*} \rightarrow \Gamma^{*}$ be the homomorphism mapping each element of $\Theta$ to the corresponding word over $\Gamma$. Consider then some word $x \in D$ of length $n$ and its image $v=g(x) \in C$. Since $g$ is length-monotonic $|v|=G_{g}(|x|)=G_{g}(n)$ and we can factorise $v$ as $v_{1} v_{2} \cdots v_{n}$ where $\left|v_{i}\right|=\partial G_{g}[i]$ for each $i \leq n$.

We define the mapping $\tau: C \rightarrow \Theta^{*}$ by setting for each $v \in C$ with factorisation $v=v_{1} v_{2} \ldots v_{n}$ as above $\tau(v)=v_{1} \cdot v_{2} \cdot \ldots \cdot v_{n}$ when considered as a word of length $n$ over $\Theta$. In particular, $\beta \circ \tau$ is the identity map on $C$. Finally, we set $h=\tau \circ g$. Thus, $h$ is by definition length-preserving.

It remains to show that $\tau$ is a semi-synchronously rational transduction. Lemma 3 tells us that the sequence of increments, $\partial G_{g}$, is ultimately periodic, say from threshold $N$ and with period $p$. Let $q=G_{g}(N+p)-G_{g}(N)$ be the total length of any $p$ consecutive blocks (increments) with sufficiently high indices. This means that after reading the first $G_{g}(N)$ input symbols and the first $N$ output symbols a transducer accepting $\tau$ can proceed by reading blocks of $q$ input symbols over $\Gamma$ and $p$ output symbols over $\Theta$ in each step, which implies that $\tau$ is in fact a $(q, p)$-synchronous transductions.

\subsection{Main result}

In this subsection we prove that every weakly regular translation is a semi-synchronous transduction, thus establishing the converse of Corollary 2. As a first step toward our main theorem let us consider the special case of length-preserving translations. The next result shows that in this case the condition of weak regularity can be dramatically weakened to preserving regularity of only binary relations in just one direction.

Proposition 4 Let $f: D \rightarrow C$ be a length-preserving translation. If $f$ preserves regularity of all binary relations on $D$ then (the graph of) $f$ is regular. ${ }^{4}$

Proof Let $\Sigma$ be the alphabet of $D$. For each $z \in \Sigma^{*}$ we define the regular relation $S_{z}=(\Sigma \times \Sigma)^{*}\left(\{\varepsilon\} \times z \Sigma^{*}\right) \cap D^{2}$. By assumption, their images under $f$ are regular relations over $C$. In fact, since only the length of the first component plays a role in these relations, and it is preserved by $f$, the following "variants" over $D \times C$ are also regular.

$$
R_{z}=\left\{(y, f(x)) \in D \times C\left|\exists y^{\prime} \in \Sigma^{*}\right| y^{\prime}|=| y \mid \wedge y^{\prime} z \preceq_{\text {prefix }} x\right\} \quad\left(z \in \Sigma^{*}\right)
$$

Indeed, an automaton for $R_{z}$ needs only to guess $v \in C$ of length $|v|=|y|$ and check that $(v, f(x)) \in f\left(S_{z}\right)$.

Since the claim is trivially true if $D$ is finite, we may assume that $D$ is infinite. Then there is a $M$ such that for every $n \in \mathbb{N}$ there is a word $y \in D$ of length $n \leq|y|<n+M$. Observe that thus every $x \in D$ is completely determined by the set of pairs $(|y|, z)$

\footnotetext{
4 Cf. [8, Corollary 6.6] (also [9]) stating that length-preserving rational transductions are synchronised rational.
} 
with $|z| \leq M$ and such that $S_{z}(y, x)$ holds. We can therefore describe $f$ using relations $R_{z}$ with $|z| \leq M$ as follows.

$$
\operatorname{graph}(f)=\left\{(x, u) \in D \times C|| x|=| u \mid \wedge \forall y \in D \bigwedge_{z \in \Sigma \leq M} S_{z}(y, x) \rightarrow R_{z}(y, u)\right\}
$$

This shows that the graph of $f$ is indeed regular, i.e. that $f$ is a synchronised rational transduction.

In the general case the lemmata of the previous subsection allow us to successively weaken the condition of weak regularity, each in turn equivalent to semi-synchronicity.

Theorem 2 For every translation $f: D \rightarrow C$ the following are equivalent:

1) $f$ is weakly regular;

2) $f$ is regularity preserving and $f^{-1}(\mathbb{L})$ is regular;

3) $f(R)$ is regular for every binary regular relation $R$ and $f$ has bounded delay;

4) $f$ can be decomposed as $f=\rho \circ h$, where $h$ is a length-preserving synchronised rational translation, $f \sim h$ and $\rho$ is a semi-synchronously rational transduction witnessing this equivalence;

5) $f$ is a semi-synchronously rational transduction.

Proof Assuming 4), $f$ is the composition of semi-synchronously rational transductions. Therefore, as noted on page $3, f$ is itself semi-synchronous. This proves 4$) \Longrightarrow 5$ ). The implication 5) $\Longrightarrow 1$ ) is just a restatement of Corollary 2 established in Proposition 2. By definition we have that 1) $\Longrightarrow 2$ ) and by Lemma 1 also 2) $\Longrightarrow$ 3) holds.

It remains to prove 3) $\Longrightarrow$ 4). We achieve this by constructing in two steps of equivalent transformations a length-preserving translation $h$ equivalent to $f$. It follows then that, like $f, h$ preserves the regularity of all binary relations. Hence by Proposition 4 it is synchronised rational as required.

As a first step we apply Lemma 2 to transform $f$ into the equivalent lengthmonotonic translation $g=\pi \circ f$. In Lemma 2 we have pointed out that $\pi$ is indeed synchronised rational and also that $g$ has bounded delay, because $f$ does. The next transformation step producing a length-preserving translation $h=\tau \circ g$ equivalent to $g$ and with $\tau$ a semi-synchronously rational translation is facilitated by Lemma 4 .

Putting the pieces together we have the following chain of equivalent transformations: $f \sim g=\pi \circ f \sim h=\tau \circ g$ amounting to the decomposition of $f$ as

$$
f=\pi^{-1} \circ \tau^{-1} \circ h
$$

where $\pi$ applies the padding, $\tau$ the cutting of words into blocks, and where $h$ is lengthpreserving, hence, according to Proposition 4, also regular. Setting $\rho=\pi^{-1} \circ \tau^{-1}$ this concludes the proof of 3$) \Longrightarrow 4$ ) thus closing the loop of implications and completing the proof of Theorem 2 .

As a final note, observe that both $\pi^{-1}$ of Lemma 2 and $\tau^{-1}=\beta$ of Lemma 4 are homomorphisms, hence $\rho$ is a homomorphism as well.

We have seen how the fact that certain selected relations are mapped onto regular relations by a translation, and/or its inverse, ensures that the translation is weakly regular. We close our discussion with an observation on how the growth rate of the density of the domain of the translation can help to further reduce these requirements. 
Theorem 3 Let $D$ be an infinite regular language.

(i) If $D$ is of exponential density then every translation $f$ with domain $D$ that maps every binary regular relation $R$ over $D$ to a regular relation is in fact weakly regular.

(ii) If, on the other hand, $D$ has polynomial density then there is a translation $f$ with domain $D$ that is regularity preserving but not weakly regular. In fact, there are infinitely many pair-wise inequivalent regularity-preserving translations: $f_{p}: D \rightarrow$ $C_{p}$, one for each prime $p$.

Before giving the proof we state an important special case of item (i).

Corollary 4 Consider a non-unary alphabet $\Sigma$ and a translation $f: \Sigma^{*} \rightarrow C$. If $f$ preserves regularity of all binary relations over $\Sigma^{*}$ then $f$ is weakly regular, and hence semi-synchronous.

Proof of Theorem 3

(i) In light of Lemma 2 we may assume that $f$ is length-monotonic and according to Theorem 2 item 3 ) we only need to show that $f$ has bounded delay.

Consider the regular relation $S=\left\{(x, y) \in D^{2}|| x|+1 \geq| y \mid\right\}$. By assumption $f(S)$ is regular, and because it is locally finite we find a constant $K$ such that $|f(y)| \leq$ $|f(x)|+K$ for all $x$ and $y$ with $|x|+1 \geq|y|$. Thus, $G_{f}(n) \leq G_{f}(n-1)+K$ for every $n$.

Let $d$ be the density function of $D$ mapping each $n$ to $d(n)=\left|D_{\leq n}\right|$. By Fact 5 there is an $\alpha>1, r \in \mathbb{N}$ and $c_{1}, c_{2}>0$ and $N \in \mathbb{N}$ such that $c_{1} n^{r} \alpha^{n} \leq \bar{d}(n) \leq c_{2} n^{r} \alpha^{n}$ for all $n \geq N$. Now suppose that for some $n>N$ and $t>0$ we find the following situation.

$$
G_{f}(n-1)<G_{f}(n)=G_{f}(n+1)=\ldots=G_{f}(n+t-1)<G_{f}(n+t)
$$

By the choice of $K$ we have that $C_{\leq G_{f}(n)} \subseteq C_{\leq G_{f}(n-1)+K}$. From Fact 4 we know that $\left|C_{<n+K}\right| \in \mathcal{O}\left(\left|C_{<n}\right|\right)$, thus, there is a constant $B$ independent of $n$ (and certainly $B \geq 1)$ such that $\left|C_{\leq G_{f}(n)}\right| \leq\left|C_{\leq G_{f}(n-1)+K}\right| \leq B \cdot\left|C_{\leq G_{f}(n-1)}\right|$. Because $f$ is length-monotonic we have $\left|C_{\leq G_{f}(n-1)}\right|=d(n-1)$ and $\left|C_{\leq G_{f}(n)}\right|=\left|C_{\leq G_{f}(n+t-1)}\right|=$ $d(n+t-1)$ since these sets contain precisely the images of words of length at most $n-1$ and $n+t-1$, respectively. Our estimates on $d(n)$ yield that

$$
\frac{c_{1}}{c_{2}} \alpha^{t} \leq \frac{c_{1}(n+t)^{r}}{c_{2} n^{r}} \alpha^{t} \leq \frac{d(n+t-1)}{d(n-1)} \leq B
$$

therefore $t \leq \log _{\alpha}\left(B \frac{c_{2}}{c_{1}}\right)$. It follows that $f$ has bounded delay, with bound equal to the maximum of $\log _{\alpha}\left(B \frac{c_{2}}{c_{1}}\right)$ and the largest $t$ such that $G_{f}(n)=G_{f}(n+t-1)$ for some $n \leq N$.

(ii) As noted in Fact 5 regular sets of polynomial growth are characterised as those being a finite (wlog. disjoint) union of the form $D=\bigcup_{i=1}^{N} u_{i, 1} v_{i, 1}^{*} u_{i, 2} \ldots u_{i, n_{i}} v_{i, n_{i}}^{*} u_{i, n_{i}+1}$. Let $n=\max _{i \leq N} n_{i}+1$. The idea is to first represent each

$$
w=u_{i, 1} v_{i, 1}^{r_{1}} \cdot u_{i, 2} v_{i, 2}^{r_{2}} \cdots \cdot u_{i, n_{i}} v_{i, n_{i}}^{r_{n_{i}}} \cdot u_{i, n_{i}+1}
$$

by the $(n+1)$-tuple of naturals

$$
t(w)=\left(i,\left|u_{i, 1} v_{i, 1}^{r_{1}}\right|,\left|u_{i, 1} v_{i, 1}^{r_{1}} u_{i, 2} v_{i, 2}^{r_{2}}\right|, \ldots,\left|u_{i, 1} v_{i, 1}^{r_{1}} \ldots u_{i, n_{i}} v_{i, n_{i}}^{r_{n_{i}}}\right|,|w|, \ldots,|w|\right) .
$$


This is an injective mapping $t: D \rightarrow \mathbb{N}^{n+1}$. In order make our case we further define a kind of unary encoding of each tuple $t(w)=\left(i, m_{1}, m_{2}, \ldots, m_{n}\right)$ as

$$
h(w)=i a_{1}^{l_{1}} a_{2}^{l_{2}} \cdots a_{n_{i}}^{l_{n_{i}}} a_{n_{i}+1}^{l_{n_{i}+1}}
$$

where $l_{1}=m_{1}$ and $l_{j+1}=m_{j+1}-m_{j}$ for each $j \leq n_{i}$. That is, $l_{j}=\left|u_{i, j} v_{i, j}^{r_{j}}\right|=$ $\left|u_{i, j}\right|+r_{j}\left|v_{i, j}\right|$ for each $j \leq n_{i}$ and $l_{n_{i}+1}=\left|u_{i, n_{i}+1}\right|$.

Claim The mapping $h: D \rightarrow \bigcup_{i=1}^{N} i a_{1}^{*} a_{2}^{*} \cdots a_{n_{i}}^{*}$ is a synchronised rational translation.

Proof Clearly, $h$ is injective, and it is easily seen to be computable by a transducer, which, after reading and storing the initial symbol $i$ of the second tape, proceeds in a letter-by-letter fashion. The transducer simply matches, for $j=1, \ldots, n_{i}+1$, each maximal factor $a_{j}^{l_{j}}$ of the second tape with a factor $u_{i, j} v_{i, j}^{r_{j}}$ in the corresponding position and of the same length on the first tape.

Claim The function $t: D \rightarrow \mathbb{N}^{n+1}$ maps every regular $R \subseteq D^{r}$ to a Presburgerdefinable subset of $\mathbb{N}^{(n+1) r}$.

Proof Notice that if one identifies each letter $a_{j}$ with $\square^{j-1} 1^{n-j} \in\{1, \square\}^{n}$ then for each word $w \in D$ with $t(w)=\left(i, m_{1}, \ldots, m_{n}\right) \in \mathbb{N}^{n+1}$ the sequence $a_{1}^{l_{1}} a_{2}^{l_{2}} \cdots a_{n_{i}+1}^{l_{n_{i}+1}}$ of $h(w)$ without the initial $i$ corresponds exactly to the letter-by-letter convolution of the unary numerals $1^{m_{1}}, \ldots, 1^{m_{n}}$ representing the tuple $t(w)$ without the $i$. This means that $h(R)$ is essentially identical, modulo the encoding of $i$, to the unary representation $[t(R)]_{1}$ of $t(R)$ for every relation $R$ over $D$. From the previous claim we know that $R$ is regular iff $h(R)$ is regular, which is, by the above, equivalent to $[t(R)]_{1}$ being regular, which in turn implies that $t(R)$ is Presburger definable (cf. footnote 2 ).

Let $[n]_{p}$ denote the $p$-ary representation of $n$ in least-significant digit first manner. For each prime $p$ we define $f_{p}$ to map each word $w \in D$ with $t(w)=\left(i, m_{1}, m_{2}, \ldots, m_{n}\right)$ to the letter-by-letter convolution

$$
f_{p}(w)=\bigotimes_{\mathbf{1}}\left([i]_{p},\left[m_{1}\right]_{p},\left[m_{2}\right]_{p}, \ldots,\left[m_{n}\right]_{p}\right)
$$

Thus, the image $f_{p}(R)$ of a relation $R \subseteq D^{r}$ is essentially identical with the natural base-p representation $[t(R)]_{p}$ of $t(R) \subseteq \overline{\mathbb{N}}^{r}(n+1)$. According to the previous claim, $t(R)$ is Presburger definable whenever $R$ is regular. As already mentioned in connection with Example 2, all Presburger-definable relations are regularly represented in the binary, or, for that matter, in any natural base- $p$ numeration system [6]. This means that each $f_{p}$ is indeed a regularity-preserving translation with domain $D$.

To conclude we observe that by the Cobham-Semenov Theorem (cf. e.g. $[6,5,12]$ ) the $f_{p}$ 's are pair-wise inequivalent. Alternatively, this can be established using Theorem 2 together with Theorem 1 showing that if $D$ is infinite and $p$ and $q$ are distinct primes then the translation $f_{q} \circ f_{p}^{-1}$ is not semi-synchronously rational.

Acknowledgements I thank the anonymous referee for his or her uncompromising critique, scrutiny, and valuable suggestions, in particular, for the idea of a simplified proof of Proposition 3. 


\section{References}

1. V. Bárány. Invariants of automatic presentations and semi-synchronous transductions. In STACS '06, volume 3884 of $L N C S$, pages 289-300, 2006.

2. M.-P. Béal, S. Lombardy, and J. Sakarovitch. Conjugacy and equivalence of weighted automata and functional transducers. In $C S R$, pages 58-69, 2006.

3. M.-P. Béal and D. Perrin. Symbolic Dynamics and Finite Automata. In A. Salomaa and G. Rosenberg, editors, Handbook of Formal Languages, Vol. 2, pages 463-503. Springer Verlag, 1997.

4. J. Berstel. Transductions and Context-Free Languages. Teubner, Stuttgart, 1979.

5. A. Bés. An Extension of the Cobham-Semënov Theorem. J. of Symb. Logic, 65(1):201-211, 2000.

6. V. Bruyère, G. Hansel, Ch. Michaux, and R. Villemaire. Logic and p-recognizable sets of integers. Bull. Belg. Math. Soc., 1:191 - 238, 1994.

7. O. Carton. The growth ratio of synchronous rational relations is unique. In Developments in Language Theory, volume 4036 of $L N C S$, pages 270-279, 2006.

8. C. C. Elgot and J. E. Mezei. On relations defined by generalized finite automata. IBM J. Research and Development, 9:47 - 68, 1965.

9. Ch. Frougny and J. Sakarovitch. Synchronized rational relations of finite and infinite words. Theoretical Computer Science, 108:45-82, 1993.

10. B. Khoussainov, A. Nies, S. Rubin, and F. Stephan. Automatic structures: Richness and limitations. In $L I C S$, pages 44-53, 2004.

11. H. A. Mauer and M. Nivat. Rational bijection of rational sets. Acta Informatica, 13:365378,1980 .

12. A. A. Muchnik. The definable criterion for definability in Presburger arithmetic and its applications. Theor. Comput. Sci., 290(3):1433-1444, 2003.

13. A. A. Nabebin. Expressibility in a restricted second-order arithmetic. Siberian Mathematical Journal, 18(4):588-593, 1977. DOI: 10.1007/BF00967200.

14. J.-E. Pin and J. Sakarovitch. Une application de la representation matricielle des transductions. Theor. Comput. Sci., 35:271-293, 1985.

15. J.-E. Pin and P. V. Silva. A topological approach to transductions. Theoretical Computer Science, 340(1):443-456, 2005

16. G. Păun and A. Salomaa. Thin and slender languages. Discrete Applied Mathematics, 61(3):257-270, 1995

17. J. Sakarovitch. Éléments de théorie des automates. Vuibert, 2003.

18. A. Salomaa and M. Soittola. Automata-Theoretic Aspects of Formal Power Series. Springer-Verlag, 1978.

19. A. Szilard, Sh. Yu, K. Zhang, and J. Shallit. Characterizing regular languages with polynomial densities. In $M F C S$, volume 629 of $L N C S$, pages 494-503, 1992. 